\title{
An Ancient Chinese Herbal Decoction Containing Angelicae Sinensis Radix, Astragali Radix, Jujuba Fructus, and Zingiberis Rhizoma Recens Stimulates the Browning Conversion of White Adipocyte in Cultured 3T3-L1 Cells
}

\author{
Guowei Gong, ${ }^{1}$ Guangyi Han, ${ }^{2}$ Huan He, ${ }^{1}$ Tina T. X. Dong $\mathbb{D}^{3,4}$ \\ Karl W. K. Tsim $\mathbb{D}^{3,4}$ and Yuzhong Zheng $\mathbb{D}^{5}$ \\ ${ }^{1}$ Department of Bioengineering, Zunyi Medical University, Zhuhai Campus, Zhuhai, Guangdong, 519041, China \\ ${ }^{2}$ Gansu Institute for Drug Control, Lanzhou, Gansu, 730070, China \\ ${ }^{3}$ Shenzhen Key Laboratory of Edible and Medicinal Bioresources, SRI, The Hong Kong University of Science and Technology, \\ Shenzhen, 518057, China \\ ${ }^{4}$ Division of Life Science, Center for Chinese Medicine, The Hong Kong University of Science and Technology, \\ Clear Water Bay, Hong Kong \\ ${ }^{5}$ Department of Biology, Hanshan Normal University, Chaozhou, Guangdong 521041, China
}

Correspondence should be addressed to Karl W. K. Tsim; botsim@ust.hk and Yuzhong Zheng; zhengyuzhong@gmail.com

Received 6 March 2019; Revised 29 May 2019; Accepted 4 June 2019; Published 16 June 2019

Guest Editor: Chongshan Dai

Copyright (C) 2019 Guowei Gong et al. This is an open access article distributed under the Creative Commons Attribution License, which permits unrestricted use, distribution, and reproduction in any medium, provided the original work is properly cited.

Background. Abnormal storage of white adipocyte tissue (WAT) is the major factor causing obesity. The promising strategies for obesity treatment are building up the brown adipocyte tissue (BAT) and/or expedite fatty acid catabolism. Traditional Chinese Medicine (TCM) sheds light on preventing obesity. Ginger is one of the most effective herbs for antiobesity by accelerating browning WAT. To fortify the antiobesity effect of ginger, an ancient Chinese herbal decoction composed of four herbs, Angelicae Sinensis Radix (ASR), Astragali Radix (AR), Jujuba Fructus (JF), and Zingiberis Rhizoma Recens (ZRR; ginger), was tested here: this herbal formula was written in AD 1155, named as Danggui Buxue Tang $\left(\mathrm{DBT}_{1155}\right)$. Therefore, the antiobesity function of this ancient herbal decoction was revealed in vitro by cultured 3T3-L1 cells. Materials and Method. The lipid accumulation was detected by Oil Red $\mathrm{O}$ staining. Furthermore, the underlying working mechanisms of antiobesity functions of $\mathrm{DBT}_{1155}$ were confirmed in $3 \mathrm{~T} 3-\mathrm{L} 1$ cells by confocal microscopy, western blot, and RT-PCR. Results. DBT $_{1155}$ was able to actuate brown fat-specific gene activations, which included (i) expression of PPAR $\gamma, \mathrm{UCP} 1$, and PCG1 $\alpha$ and (ii) fatty acid oxidation genes, i.e., CPT1A and HSL. The increase of browning WAT, triggered by $\mathrm{DBT}_{1155}$, was possibly mediated by a $\mathrm{Ca}^{2+}$-AMPK signaling pathway, because the application of $\mathrm{Ca}^{2+}$

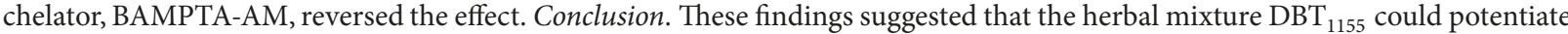
the antiobesity functions of ginger, which might have potential therapeutic implications.

\section{Introduction}

Obesity is characterized as abnormal or excessive accumulated adipose tissues, which is believed to be induced by multiple factors, including genetically and environmentally. Obesity incidence is increasing and becomes a normal phenomenon in both developing and developed countries, posting a great challenging for health care professionals.
The obese persons could undergo high risks of metabolic abnormalities, diabetes, and several types of cancers diseases $[1,2]$. Antiobesity therapeutic treatments have been proposed for decades. The limitation of carbohydrate intake used to be believed as the most effective strategy for antiobesity; however, this treatment has been reported to have negative impact on mental development $[3,4]$. On the other hand, the side effects of popular weight loss synthetic medicines, 
e.g., phentermine-topiramate and lorcaserin, are commonly ameliorating the risks of hepatorenal syndrome and resulting in reducing the patient's life quality [5].

There are two types of adipose tissues found within human body, i.e., white adipose tissues (WAT) and brown adipose tissues (BAT). The major functions of WAT are heating insulation, buffering mechanical cushion, and, finally, storing of energy. WAT is acting as fuel for energy imbalances when the intaking energy is smaller than outputting energy; therefore, WAT is considered as a crucial component in contributing obesity [6]. BAT, on the other hand, accelerates energy expenditure and finally combats obesity $[7,8]$. Physical exercise is one of typical routines to lose weight and reshape the body by hastening WAT browning and stimulating fatty acid oxidation [9]. The high expression level of mitochondrial uncoupling protein 1 (UCP1) is a hallmark of browning WAT [9]. Furthermore, peroxisome proliferatoractivated receptor $(\operatorname{PPAR} \gamma)$ and peroxisome proliferatoractivated receptor-gamma coactivator 1 (PGCl $\alpha)$ are two transcriptional factors in modulating adipogenous-related gene expressions, which are highly expressed in BAT [10]. On the other hand, carnitine palmitoyl transferase I A (CPT1A) and hormone-sensitive lipase (HSL) genes can enhance mitochondrial activities and stimulate fatty acid oxidation, and therefore they are classified as the signature of fatty acid oxidation [11].

There is an increasing consumption of functional foods or food supplements aiming to control weight. Traditional Chinese Medicine (TCM) has drawn attention in the market; because it has a peculiar and organized understanding of obesity according to its specific concept. The major determinant eliciting obesity is the imbalance of "Yin" and "Yang," which results in stagnation of "Qi" and "Blood" [12]. Most of synthetic medicines alter the interaction of neurological and/or hormonal signals in acting as appetite suppressants or as inducers of diarrhea, which could be damaging to psychology and physiology of our bodies [13]. Zingiberis Rhizoma Recens (ZRR, root of Zingiber officinale Roscoe; ginger) is one of the most popular spices utilized in the world, and its antiobesity function has been widely reported [14]. The intake of ginger extract or curcumin, one of bioactive constituents found within ginger, significantly reduced body weight, leptin, insulin, amylase, lipase plasma, and tissue lipids in rats. In parallel, the level of peroxisomal catalase in serum was enhanced in ginger- and curcumin-treated rats [15-18]. ZRR was able to activate AMPK pathway, the key signaling in modulating WAT browning [19]. On the other way, oral administration of ZRR in human could reduce hunger sensation [15]. In TCM formulation, ginger is being included commonly in many herbal formulae, and indeed the therapeutic functions of these herbal formulae are believed to enhance thermogenesis, as such to reduce obesity.

An ancient herbal mixture, written by Chen Suan of Song Dynasty (1155 AD) in "Chensuan Fuke Buji", is known to improve "Qi" and "Blood," named as Danggui Buxue Tang $\left(\mathrm{DBT}_{1155}\right) \cdot \mathrm{DBT}_{1155}$ composes four herbs: Angelicae Sinensis Radix (ASR), Astragali Radix (AR), Jujuba Fructus (JF), and Zingiberis Rhizoma Recens (ZRR) in a weight ratio of 36: 30: 15: 20. The antiobesity functions of curcumin-enriched ZRR were widely reported, and this herbal formula was shown to have antilipid accumulation in our preliminary study. Thus, the antiobesity functions of $\mathrm{DBT}_{1155}$ in cultured 3T3$\mathrm{L} 1$ adipocytes were tested here.

\section{Materials and Methods}

2.1. Preparation of Herbal Extract. The raw herbs of root of Astragali membranaceus var. mongholicus (AR), root of Angelica sinensis (Oliv) Diels. (ASR), fruit of Ziziphus jujuba $\mathrm{cV}$. Jinsixiaozao (JF), and rhizome of Zingiber officinale Roscoe (ZRR; ginger) were collected and identified in 2013. The voucher specimen of AR, ASR, JF, and ZRR was kept in Centre for Chinese Medicine of HKUST. AR, ASR, JF, and ZRR in a weight ratio of 36: 30: 15: 20 were used to prepare $\mathrm{DBT}_{1155}$ decoction. The mixture was boiled in 8 volumes of water for twice. Fifty grams of ZRR was also boiled in water twice, each with 8 volumes of water. This preparation was verified in previous studies [20, 21]. All samples were dried by lyophilization and resuspended in water at final concentration of $100 \mathrm{mg} / \mathrm{mL}$, which were kept at $-80^{\circ} \mathrm{C}$.

\subsection{HPLC Analysis and Chemical Quantifications. Chemical} standardization and quantification of herbal mixture are the first step in performing biological assay [20, 22, 23]. According to China Pharmacopeia, ferulic acid was chosen as marker chemical in ASR. Calycosin and formononetin were selected as quantification markers in AR; cyclic AMP (cAMP) was reported to be the bioactive chemical found within JF; and 6-gingerol was elite as standard for ZRR (CP, 2015). The HPLC mobile phases were composed of $0.1 \%$ formic acid in water (A) and $0.1 \%$ formic acid in acetonitrile (B), respectively. An elution gradient was set up as follows: 02 min isocratic gradient 95\% (A); 2-4 min, linear gradient 9590\% (A); 4-15 min, linear gradient $90-80 \%$ (A); 15-20 min, isocratic gradient $80 \%(\mathrm{~A})$; $20-27 \mathrm{~min}$, linear gradient $80 \%$ $70 \%$ (A); and $17-70 \mathrm{~min}$, linear gradient $70-45 \%$ (A). The preequilibration period of $15 \mathrm{~min}$ was used between each run. The column temperature was set to $25^{\circ} \mathrm{C}$. The injection volume was $10 \mu \mathrm{L}$. A wavelength of $254 \mathrm{~nm}$ was employed for detection. The flow rate was set at $1.0 \mathrm{~mL} / \mathrm{min}$. Agilent RRLC 1200 series system (Waldron, Germany) equipped with a degasser, a binary pump, an auto-sampler, a diode array detector (DAD), and a thermo-stated column compartment was adopted for establishment of fingerprint for herbal extracts. The HPLC condition was conducted on Agilent ZORBAX SB-Aq $(4.6 \times 250 \mathrm{~mm}, 5 \mu \mathrm{m}) \mathrm{C} 18$ column.

2.3. Cell Cultures. Mouse 3T3-L1 fibroblast cells (CL-173) were obtained from ATCC (Manassas, VA) and maintained at $37^{\circ} \mathrm{C}$ in a water-saturated incubator containing $5 \% \mathrm{CO}_{2}$ and in DMEM supplemented with $4.5 \mathrm{~g} / \mathrm{L}$ glucose, $10 \% \mathrm{FBS}$, $100 \mathrm{U} / \mathrm{mL}$ penicillin, and $100 \mu \mathrm{g} / \mathrm{mL}$ streptomycin. Induction of lipogenic differentiation was detailed in a previous study [24]. Briefly, cultured cells were treated with dexamethasone ( $1 \mu \mathrm{M}$, Sigma-Aldrich, St Louis, MO), insulin (1.8 $\mu \mathrm{M}$, SigmaAldrich), and dibutryl-cAMP (300 $\mu \mathrm{M}$, Sigma-Aldrich) for 72 hours to induce lipogenesis. The cultures were set as day 0 and replaced with the culture medium containing insulin 
$(1.8 \mu \mathrm{M})$ for every two days. At day 10 , about $80 \%$ of cultures were induced to contain triglyceride. Treatments including negative control ( $0.02 \%$ DMSO only), cocktail $(1.8 \mu \mathrm{M}$ of rosiglitazone and triiodothyronine), low concentration of DBT (DBT-L, $0.125 \mathrm{mg} / \mathrm{mL}$ ), and high concentration of DBT (DBT-H, $1.0 \mathrm{mg} / \mathrm{mL}$ ) were given to differentiated cultures (on day 10) for 72 hours. Unless described otherwise, all the culture reagents were purchased from Invitrogen Technologies (Waltham, MA).

2.4. Cell Viability. The cell viability was measured by MTT assay. In brief, cells were cultured in 96-well plate. After drug treatments for indicated durations, MTT solution was added into the cultures in the final concentration of $0.5 \mathrm{mg} / \mathrm{mL}$; after incubation for 2 hours, the production of purple crystal was dissolved by DMSO solvent. The absorbance at $570 \mathrm{~nm}$ was measured.

2.5. Oil Red O Staining. Oil Red O at $0.2 \%$ in isopropanol was filtered. Experimental cultured cells were washed with PBS, fixed by paraformaldehyde (4\% in PBS, Sigma-Aldrich) for $5 \mathrm{~min}$, incubated with Oil Red $\mathrm{O}$ staining for $30 \mathrm{~min}$, and washed twice with PBS. The stained triglyceride (TG) was resolved in isopropanol and measured at the absorbance of $490 \mathrm{~nm}[24]$.

2.6. Laser Confocal Fluorescence Microscopy. Fluorimetric measurements were performed on cultured 3T3 cells using an Olympus Fluoview FV1000 laser scanning confocal system (Olympus America, Manassas, VA) mounted on an inverted Olympus microscope, equipped with a $10 \mathrm{X}$ objective. Intracellular $\mathrm{Ca}^{2+}$ concentration was detected by fluorescent calcium indicator Fluo-4 AM (Sigma-Aldrich). Cultured cells were seeded on the glass coverslips and incubated for $30 \mathrm{~min}$ at $37^{\circ} \mathrm{C}$ in a normal physiological solution containing $\mathrm{Ca}^{2+}$ free normal physiological solution containing $5 \mu \mathrm{M}$ Fluo- 4 AM. A23187 (Sigma-Aldrich), a calcium ionophore, was used as a positive control. The amount of $\mathrm{Ca}^{2+}$ was evaluated by measuring the fluorescence intensity exiting at $488 \mathrm{~nm}$ and emitted at $525 \mathrm{~nm}$.

2.7. Western Blot Assay. The protein expressions of PPAR $\gamma$, PGC1 $\alpha$, UCP1, and internal control GAPDH were revealed by western blot. Cultures were seeded onto 6-well plate. After drug treatment for 72 hours, including inhibitor application, the cultures were harvested in high salt lysis buffer $(1 \mathrm{M}$ $\mathrm{NaCl}, 10 \mathrm{mM}$ HEPES, pH 7.5, 1 mM EDTA, 0.5\% Triton X100 ), followed by centrifugation at $16,100 \mathrm{rpm}$ for $10 \mathrm{~min}$ at $4^{\circ} \mathrm{C}$. Samples with equal amount of total protein were added with $2 \mathrm{X}$ lysis buffer $(0.125 \mathrm{M} \mathrm{HCl}, \mathrm{pH} 6.8,4 \%$ SDS, $20 \%$ glycerol, 2\% 2-mercaptoethanol and $0.02 \%$ bromophenol blue) and heated to $95^{\circ} \mathrm{C}$, and the protein was subjected to SDS-PAGE analysis. After transferring, the membranes were incubated with antibodies against PPAR $\gamma, \mathrm{PGC} 1 \alpha$, UCP1, and GAPDH (CST, Danvers, MA) at 1: 3,000 dilutions at cold room overnight.
The phosphorylation of AMPK was also determined by western blot assay. Differentiated cultures were serumstarved for 3 hours before the drug application. After treatment with BAMPTA-AM $(10 \mu \mathrm{M})$ or WZ4003 (100 nM; Selleck, Munich, Germany), the cultures were collected immediately in lysis buffer (125 mM Tris-HCl, 2\% SDS, 10\% glycerol, $200 \mathrm{mM}$ 2-mercaptoethanol, $\mathrm{pH}$ 6.8). The protein was subjected to SDS-PAGE analysis. After transferring the proteins to membranes, the membranes were incubated with anti-phospho-AMPK (Cell Signaling, MA) at 1: 5,000 dilution and anti-total-AMPK (Cell Signaling) at 1: 5,000 dilution at $4^{\circ} \mathrm{C}$ for 12 hours. Following incubation in horseradish peroxidase- (HRP-) conjugated anti-rabbit secondary antibodies in 1: 5,000 dilution for 3 hours at room temperature, the immune-complexes were visualized by the enhanced chemiluminescence (ECL) method (Amersham Biosciences, Piscataway, NJ). The band intensities in the control and agonist-stimulated samples, run on the same gel and under strictly standardized ECL conditions, were compared on an image analyzer, using in each case a calibration plot constructed from a parallel gel with serial dilutions of one of the samples.

2.8. RT-PCR Analysis. Total RNA was extracted from 3T3L1 adipocyte cells with RNAzol reagent (Invitrogen) according to manufacturer's instructions. RNA samples with OD260/OD280 ratio higher than 2.0 were employed for PCR. One $\mu \mathrm{g}$ of total RNA was employed for the production of cDNA, using a PCR system. The oligonucleotide primer sequence was as follows: peroxisome proliferator-activated receptor $(\operatorname{PPAR} \gamma): 5^{\prime}$-CCA GAG TCT GCT GAT CTG CG-3' and $5^{\prime}$-GCC ACC TCT TTG CTC TGA TC-3'; peroxisome proliferator-activated receptor $\gamma$ coactivator 1 (PGC1 $\alpha): 5^{\prime}$ GAC CTG GAA ACT CGT CTC CA-3 and $5^{\prime}$-AAA CTT GCT AGC GGT CCT CA-3'; carnitine palmitoyl transferase I A (CPT1A): $5^{\prime}$-GGA CAT TAT CAC CTT GTT TGG C$3^{\prime}$ and $5^{\prime}$-GGA GCA ACA CCT ATT CAT T- $3^{\prime}$; hormonesensitive lipase (HSL): $5^{\prime}$-GCG CTG GAG GAG TGT TTT T$3^{\prime}$ and $5^{\prime}$-CGC TCT CCA GTT GAA CCA AG-3'; mitochondrial uncoupling protein 1 (UCP1): $5^{\prime}$-GAT GGT GAA CCC GAC AAC TT- $3^{\prime}$ and $5^{\prime}$-CTG AAA CTC CGG CTG AGA AG- $3^{\prime}$; 18S: $5^{\prime}$-GTA ACC CGT TGA ACC CCA TT-3' and $5^{\prime}$-CCA TCC AAT CGG TAG TAG CG-3'. Transcript levels were quantified by using ${ }_{\Delta} \mathrm{Ct}$ value method, where the values of target genes were normalized by $18 \mathrm{~S}$ in the same sample at first before comparison. PCR products were analyzed by gel electrophoresis and melting curve analysis, as to confirm the specific amplification.

2.9. Statistical Analysis and Other Assays. Protein concentrations were measured by Bradford's method (Herculues, CA). Statistical tests have been done by using one-way analysis of variance. Data were analyzed by t-test and expressed as Mean \pm SEM. Statistically significant changes were classified as significant $(*)$ where $p<0.05$, more significant $(* *)$ where $p<0.01$, and highly significant $(* * *)$ where $p<0.001$ as compared with control group. 


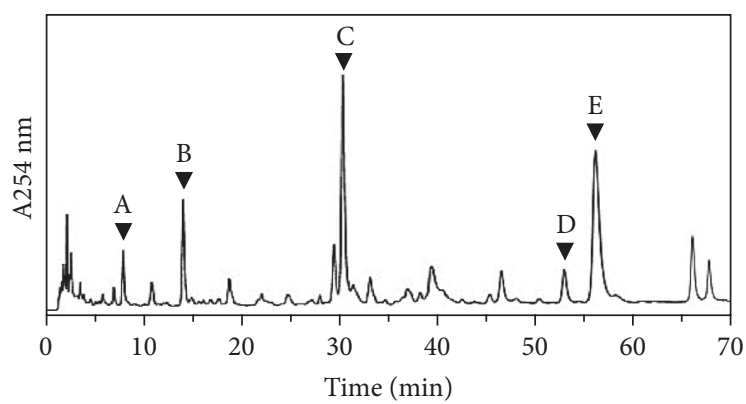

(a)

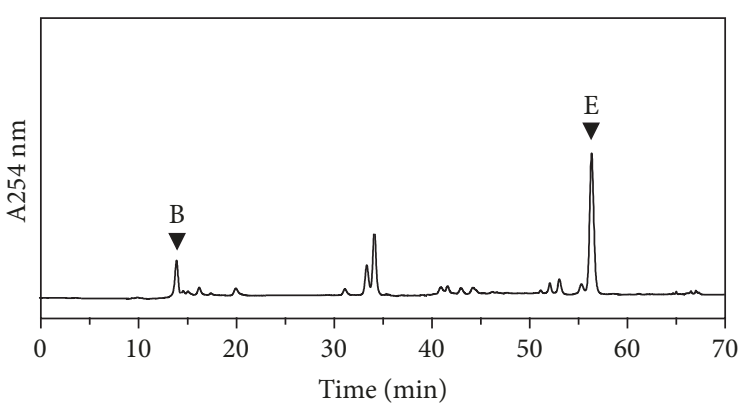

(b)

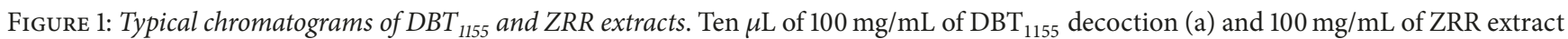
(b) were subjected to HPLC-DAD analysis, and the chemical fingerprints were revealed at the wavelength $254 \mathrm{~nm}$. The identification of ferulic acid (A), cAMP (B), calycosin (C), formononetin (D), and 6-gingerol (E) were labeled here. Representative chromatograms were shown, $n=$ 3.
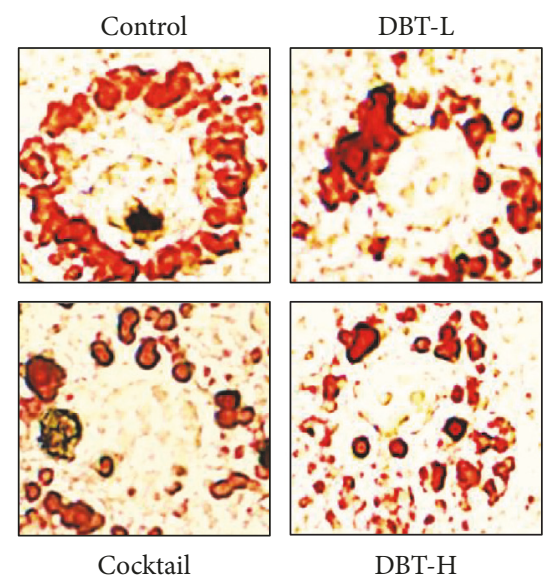

(a)

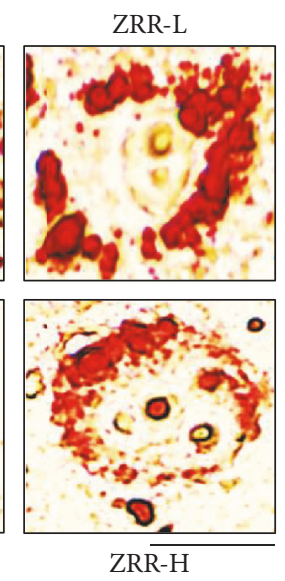

ZRR-H

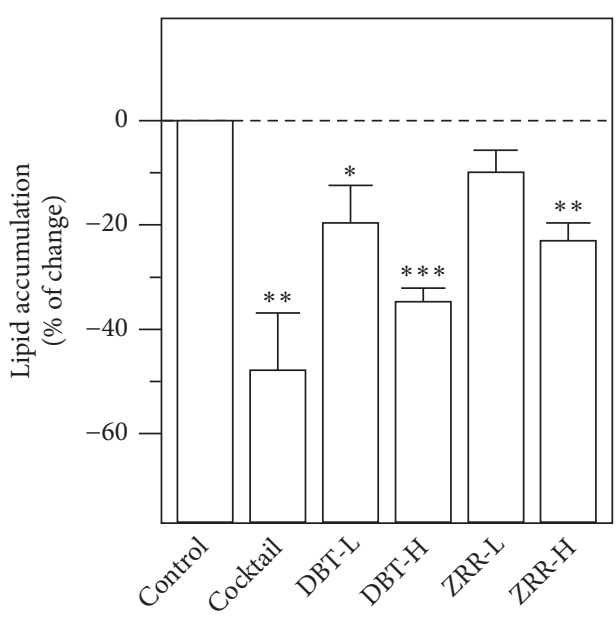

(b)

Figure 2: $D B T_{1155}$ decreases lipid accumulation. 3T3-L1 adipocytes were cultured to 10 days of differentiation and then applied with cocktail $\left(1.8 \mu \mathrm{M}\right.$ of rosiglitazone and triiodothyronine), or different concentrations of $\mathrm{DBT}_{1155}(1 \mathrm{mg} / \mathrm{mL}$ of DBT labeled as DBT-H; $0.125 \mathrm{mg} / \mathrm{mL}$ of DBT labeled as DBT-L) or ZRR (1 mg/mL of ZRR labeled as ZRR-H; $0.125 \mathrm{mg} / \mathrm{mL}$ of ZRR labeled as ZRR-L) for another 3 days. (a) Oil Red $\mathrm{O}$ staining was to measure lipid accumulation. Bar $=50 \mu \mathrm{m}$. (b) The amount of stained lipid was quantified at $490 \mathrm{~nm}$ absorbance. Data were expressed as mean \pm SEM of the percentage of change as compared with control, where $n=3 ; p<0.05(*) ; p<0.01(* *) ; p<0.001(* * *)$.

\section{Results}

3.1. Chemical Standardization of $D B T_{1155}$. Chemical standardization is to ensure the repeatability of herbal extract in all subsequent biochemical analyses. The amounts of major components were calibrated by a calibration curve derived from HPLC, which was obtained from a series of dilutions of the chemical markers. The calibration curve of ferulic acid was $y=21.134 x+19.607$; calycosin was $y=10.189 x-$ 10.129; formononetin was $y=13.602 x+12.705$; cAMP was $y=11.218 x+55.42$; and 6-gingerol was $y=17.311 x+25.1328$ (Supplementary Table 1). In quality control of herbal mixture, $1 \mathrm{~g}$ of dried $\mathrm{DBT}_{1155}$ powder was proposed to contain $572.32 \mu \mathrm{g}$ of calycosin, $205.66 \mu \mathrm{g}$ of formononetin, $150.02 \mu \mathrm{g}$ of ferulic acid, $102.35 \mu \mathrm{g}$ of cAMP, and $1296.8 \mu \mathrm{g}$ of 6-gingerol. One gram of ZRR dried extract was proposed to contain $34.63 \mu \mathrm{g}$ of cAMP and $1203.24 \mu \mathrm{g}$ of 6-gingerol. These chemical requirements set the minimal standards. In addition, HPLC fingerprint was developed for the standardized extracts at $254 \mathrm{~nm}$ wavelength (Figure 1). These chemical parameters were employed as quality control to ensure the repeatability of biochemical assays.

3.2. Browning WAT Functions. The functions of $\mathrm{DBT}_{1155}$ and ZRR on lipid accumulation of cultured 3T3-L1 adipocytes were detected by Oil Red O. The optimized working concentration of DBT was determined by MTT assay; the highest working concentration of $\mathrm{DBT}_{1155}$ should be $1 \mathrm{mg} / \mathrm{mL}$, which was labeled as DBT-H. The lowest concentration should be $0.125 \mathrm{mg} / \mathrm{mL}$ which was named as DBT-L (Supplementary Figure 1). The lipid accumulation was significantly decreased under application of $\mathrm{DBT}_{1155}$ extract, which was in a dosedependent manner (Figures 2(a) and 2(b)). One $\mathrm{mg} / \mathrm{mL}$ of DBT decoction (DBT-H) possessed $\sim 35 \%$ decrease by 


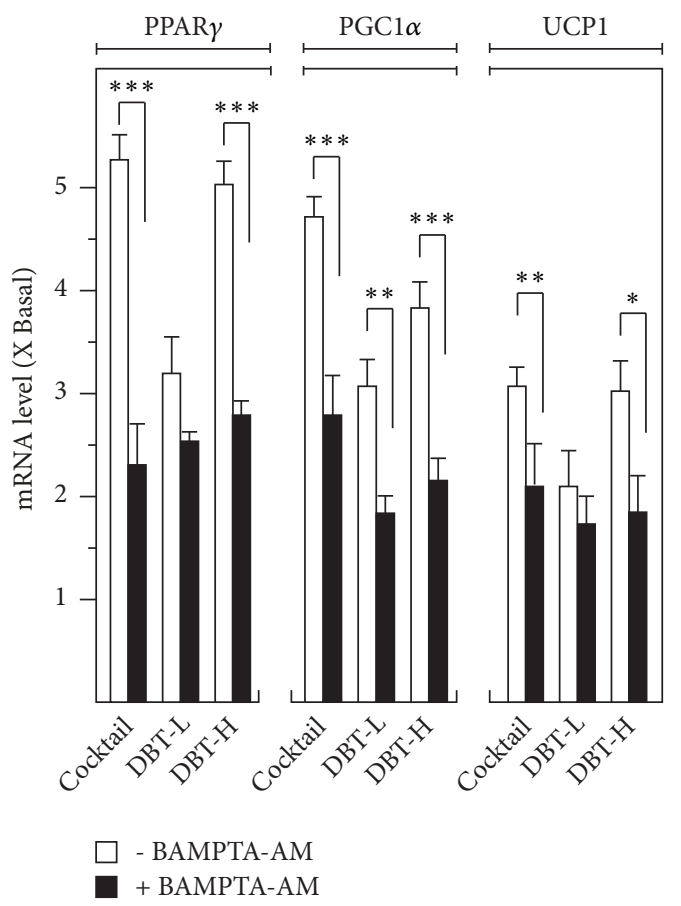

FIGURE 3: $D B T_{1155}$ triggers browning mRNA expressions of WAT markers. 3T3-L1 adipocytes were cultured to 10 days of differentiation. Then, the cultures were applied with cocktail or different concentrations of $\mathrm{DBT}_{1155}$ (DBT-H: $1 \mathrm{mg} / \mathrm{mL}$ of DBT; DBT-L: $0.125 \mathrm{mg} / \mathrm{mL})$ with/without cotreatment of BAMPTA-AM $(10 \mu \mathrm{M})$ for another 3 days. Total RNAs were isolated and reverse-transcribed to cDNA for PCR analysis. The mRNA levels of PPAR $\gamma, \mathrm{PGCl} \alpha$, and UCP1 were determined by the Ct-value method and normalized by the house keeping gene $18 \mathrm{~S}$ rRNA. Data were expressed as mean \pm SEM as compared with control, setting as 1 here, where $n=3 ; p<$ $0.05(*) ; p<0.01(* *) ; p<0.001(* * *)$.

lipid staining as compared to the negative control (Figures 2(a) and 2(b)). The antilipid accumulation effect, triggered by $\mathrm{DBT}_{1155}$, was much stronger than that of $\mathrm{ZRR}$ alone (Figures 2(a) and 2(b)). The lipid staining results implied that other constituents within $\mathrm{DBT}_{1155}$ might potentiate antilipid accumulation activity of ZRR. The $\mathrm{IC}_{50}$ of $\mathrm{DBT}_{1155}$ was $\sim 0.375 \mathrm{mg} / \mathrm{mL}$. In the same assay, the herbal extracts of AR, ASR, and JF did not show significant antilipid effect (Supplementary Figure 2). Here, the cocktail served as a positive control suppressing lipid accumulation dramatically by $\sim 50 \%$ decrease, as compared with a negative control (Figures 2(a) and 2(b)).

Increase levels of PPAR $\gamma, \mathrm{UCP} 1$, and PCG1 $\alpha$ are the hall markers of WAT browning [25]. Indeed, the activations of these genes have been reported in obesity and/or its related diseases [25]. The transcript levels of these BAT-specific genes were revealed by RT-PCR from total RNA deriving from $\mathrm{DBT}_{1155}$-treated 3T3-L1 adipocytes. As shown in Figure 3, $\mathrm{DBT}_{1155}$ increased the mRNA levels of BAT markers in a dose-dependent manner. The maximal inductions of PPAR $\gamma$, PCG1 $\alpha$, and UCP1 were revealed at $\sim 5$-fold, $\sim 4$-fold, and $\sim 3$-fold, respectively, under the application of $1 \mathrm{mg} / \mathrm{mL}$ of $\mathrm{DBT}_{1155}$. Furthermore, calcium chelator, BAMPTA-AM, was employed here to identify the signaling pathway. The pretreatment of this chelator in 3T3-L1 adipocytes dramatically suppressed the BAT-specific gene transcription (Figure 3). The protein expression levels of these markers were also taken into consideration. The translational activities of these BAT-specific genes, e.g., PPAR $\gamma$ at $\sim 58 \mathrm{kDa}$, PCG1 $\alpha$ at $~$ $100 \mathrm{kDa}$, and UCP1 at $\sim 30 \mathrm{kDa}$, were highly expressed, from 5 -to-9-fold under the challenge of $1 \mathrm{mg} / \mathrm{mL}$ of $\mathrm{DBT}_{1155}$ (Figure 4). On the other hand, the application of BAMPTAAM significantly abolished the increased protein expression, triggered by this ancient herbal formula (Figure 4). Taken together, $\mathrm{DBT}_{1155}$ decoction possessed antiobesity functions by accelerating WAT browning.

AMPK signaling is a key player in regulating browning WAT. Application of $\mathrm{DBT}_{1155}$ in cultured 3T3-L1 adipocytes was capable of inducing AMPK phosphorylation, and this activation was in a time-dependent manner (Figure 5(a) right). The maximal stimulation was shown at 2 hours, as compared to control (Figure 5(a) right). Cellular $\mathrm{Ca}^{2+}$ level has been reported to be an indispensable factor regulating AMPK activities [26]. Here, the $\mathrm{Ca}^{2+}$ concentration in the treated 3T3-L1 adipocytes was detected by confocal microscopy. Fluo-4 AM, a $\mathrm{Ca}^{2+}$ indicator, was applied onto the cultures as to monitor the variation of $\mathrm{Ca}^{2+}$ induced fluorescence signal in differentiated 3T3-L1 cells. The increased $\mathrm{Ca}^{2+}$ level was found after the treatment in 3T3-L1 adipocytes (Figure 5(a) left). A23187, a calcium ionophore, served as a positive control (Figure 5(a)). In line with the above BAT-specific gene expression results, the pretreatment of BAMPTA-AM markedly suppressed the $\mathrm{Ca}^{2+}$ influx and AMPK activation in cultured 3T3-L1 cells (Figure 5(b)). Moreover, the pretreatment with WZ4003, a specific AMPK antagonist, reduced the phosphorylation of AMPK, as shown in Figure 6. Accordingly, these data indicated that $\mathrm{DBT}_{1155}$ triggered WAT browning in adipocyte via an AMPK signaling.

3.3. Fatty Acid Catabolism Activities. The key function of fatty acid catabolism is to generate ATP, and thus fat oxidation is a key switch to reveal catabolism progress [11, 27]. CPT1A is recognized in precipitating mitochondrial activities and accelerating fatty acid oxidation [26]. The transcriptional activates of these marker genes were revealed here (Figure 7). Treatment with high dosage of $\mathrm{DBT}_{1155}(1 \mathrm{mg} / \mathrm{mL}$; DBT-H) led to significant increase of mitochondrial CPT1A mRNA, indicating the accelerated fat oxidation under the challenge of herbal decoction (Figure 7). Synthesis of fatty acid is another key regulator to modulate catabolism [26]. Overexpression of HSL was observed in the $\mathrm{DBT}_{1155}$-treated 3T3-L1 adipocytes. The maximal stimulation of HSL was revealed at $\sim 2$-fold, as compared with the control. The upregulation of HSL indicated that $\mathrm{DBT}_{1155}$ could have the possibility of suppressing fatty acid synthesis in 3T3-L1 adipocytes (Figure 7). Again, the mRNA levels of CPT1 and HSL in cultured 3T3-L1 adipocytes were downregulated upon pretreatment of BAMPTA-AM. Our data shed light on the antiobesity functions of $\mathrm{DBT}_{1155}$ via accelerating fatty acid oxidation and suppressing its synthesis. 


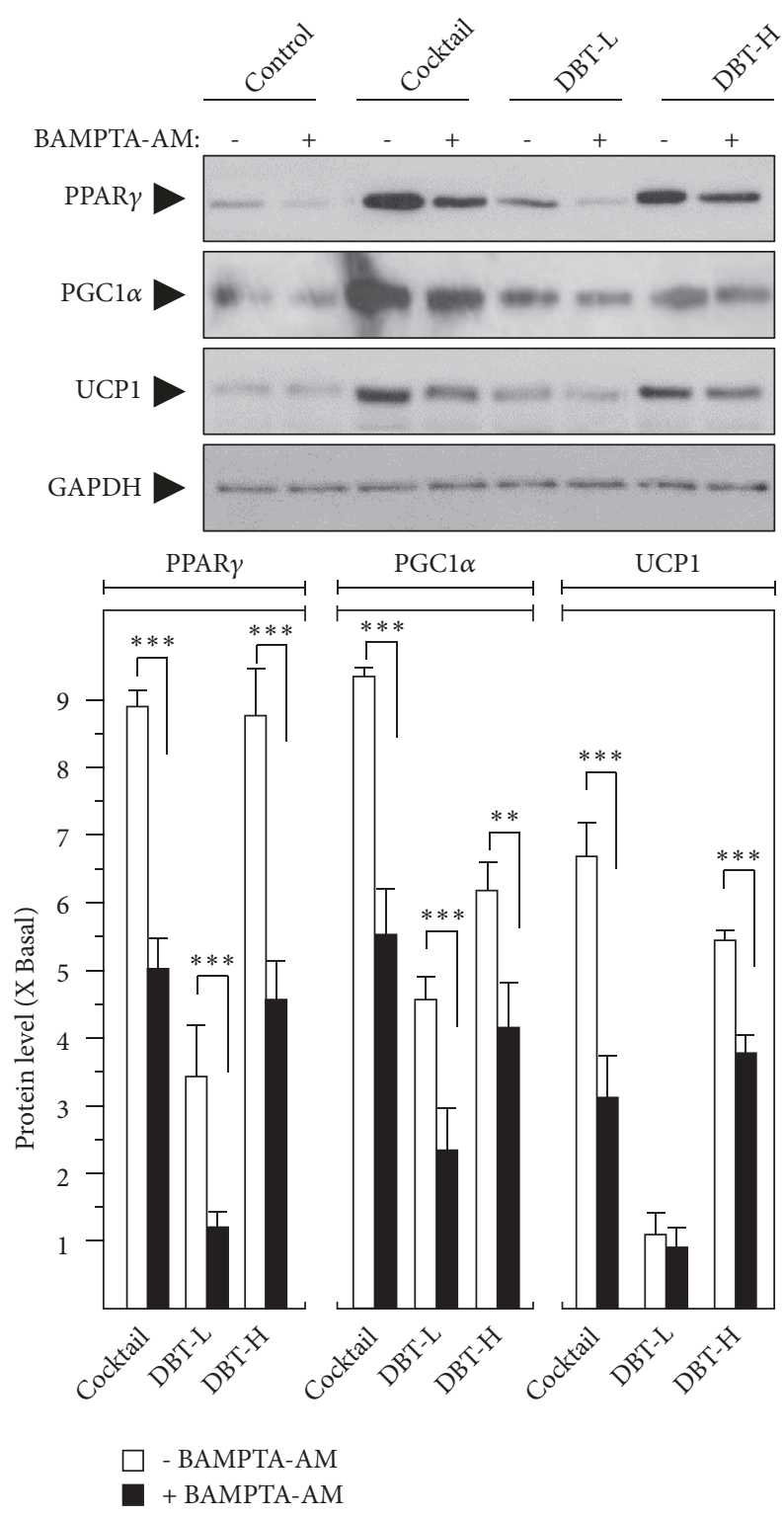

FIGURE 4: $D B T_{1155}$ triggers protein levels of browning WAT markers. 3T3-L1 adipocytes were treated with cocktail or different concentrations of DBT $_{1155}$ (DBT-H: $1 \mathrm{mg} / \mathrm{mL}$ of DBT; DBT-L: $0.125 \mathrm{mg} / \mathrm{mL}$ ) with/without cotreatment of BAMPTA-AM $(10 \mu \mathrm{M})$ for another 3 days after 10 days of differentiation, as in Figure 2. The translational levels of PPAR $\gamma(\sim 58 \mathrm{kDa}), \operatorname{PGC1} \alpha(\sim 100 \mathrm{kDa})$, and UCP1 $(\sim 30 \mathrm{kDa})$ were detected by immunoblot analysis by specific antibodies. GAPDH $(\sim 38 \mathrm{kDa})$ served as an internal control. Quantification of target protein expression was calculated by a densitometer (lower panel). Data were expressed as mean \pm SEM as compared with control, setting as 1 here, where $n=3 ; p<0.05(*) ; p<0.01(* *)$; $p<0.001(* * *)$.

\section{Discussion}

During the past decade, people paid attention to the study of weight loss therapy relying on herbal medicine. However, the side-effect of TCM in weight loss is unclear, which is one of the limitations in acceptance of TCM [21, 28].
This is an urgency to search for the safe TCM that could be effective for antiobesity. $\mathrm{DBT}_{1155}$ is a classical herbal decoction commonly used for treatment of "Blood" and "Qi" deficiencies. "Blood" in TCM theory is to provide nutrition. "Qi" is to boost antioxidative functions and finally neutralizes "Blood." Indeed, this traditional herbal formula $\mathrm{DBT}_{1155}$ is comprised of 4 herbs, and each of them plays their specific responsibilities. AR is well-known for "Qi"-reinforcing, ASR is popular for "Blood-" nourishing, JF is famous for tonifying "Qi" and "Blood," and ZRR is recognized to enhance vital energy as well as improving immune system in TCM theory [28]. $\mathrm{DBT}_{1155}$ was shown to upregulate erythropoietic genes in vitro and to reverse anemia-index in rats [21]. On the other hand, $\mathrm{DBT}_{1155}$ has been utilized for years, and the side effect has been rarely reported. Therefore, this herbal decoction should be safe to be consumed. Furthermore, the current data shed light on inducing brown fat phenotype in cultured 3T3-L1 via elevation of PPAR $\gamma$, UCP1, and PCG1 $\alpha$ in both transcriptional and translational levels.

There are three types of bioactive constituents popular for obesity treatment. The first cluster is polysaccharide isolated from plant, acting as vital role in maintaining body health [28, 29]. In obesity animal models, the polysaccharides showed the possibilities of decreasing TG level via enhancing intestinal peristalsis, upregulating lipid absorption rate, and accelerating the transformation of exogenous cholesterol to bile acids [30]. For example, the total polysaccharide extracted from ginger had dual antiobesity functions by upregulating metabolic rate and inhibiting the absorption rate of caloriedense dietary fats [31]. The JF-generated polysaccharide was capable of decreasing glycerol-3-phosphate dehydrogenase activity in vitro [32]. Flavonoid is the other bioactive group for obesity medication. Flavonoid increased thermogenesis via enhancing fatty acid transportation and reduced the triglyceride content in plasma and finally decreased lipid deposition [33]. In parallel, the in vivo working mechanism of flavonoids for losing weight has been well reported [3436]. The intake of total flavonoids showed a possibility of alleviating obesity-triggered metabolic damage via suppressing inflammation [35]. Formononetin, one of isoflavones extracted from AR, as well as in DBT, was capable of stimulating AMPK pathway in vitro, and suppressed the development of obesity by attenuating high fat diet-induced body weight gain and visceral fat accumulation [36]. The last group of nature product for obesity treatment is believed to be polyphenols. Among them, the most famous one is curcumin [37-40]. Pan et al. demonstrated that treatment of male C57BL/6 J obese mice with curcumin significantly decreased body weight and fat mass after 2 months of observation, but enhanced insulin sensitivity in mice [37]. Moreover, oral administration of curcumin-enriched supplementation was effective in suppressing oxidative stress via modulation of antioxidation enzymes activities, i.e., superoxide dismutase (SOD) and glutathione peroxidase (GPx) in obese patients $[39,40]$. The constituents of $\mathrm{DBT}_{1155}$ decoction, i.e., $\mathrm{AR}$, ASR, and JF, showed synergistic effects to ZRR because the antilipid functions of AR, ASR, and JF were very limited. The cAMP-induced AMPK signaling is the major mechanism for antiobesity [41-44]. The $\mathrm{DBT}_{1155}$-triggered AMPK signaling 
Control

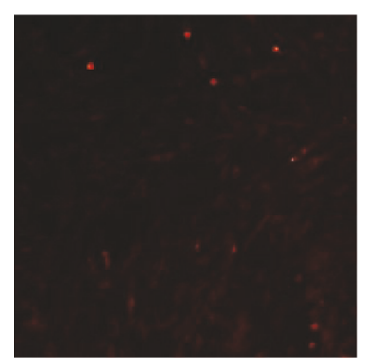

DBT-L

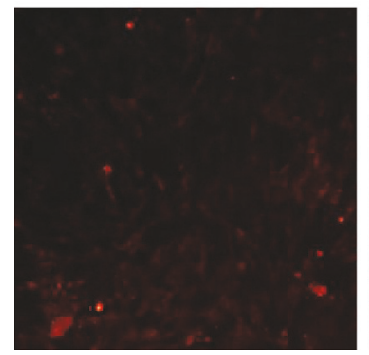

Control

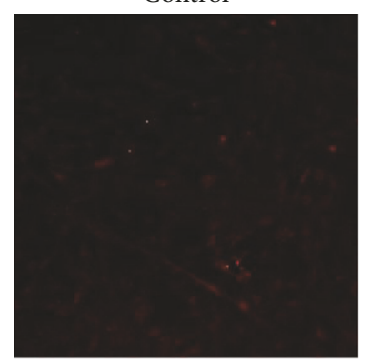

DBT-L

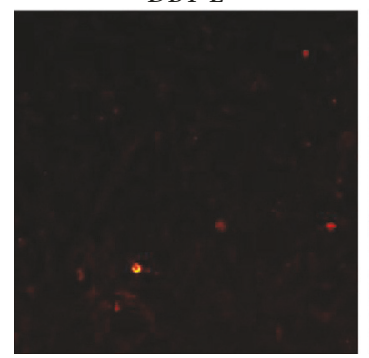

A23187

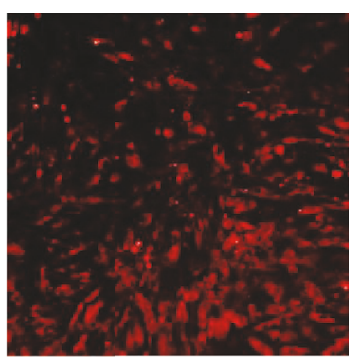

DBT-H

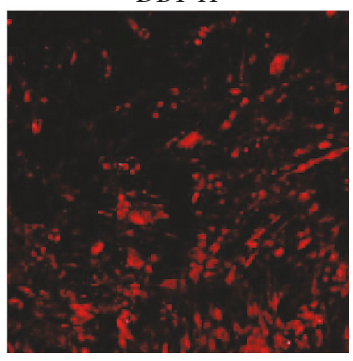

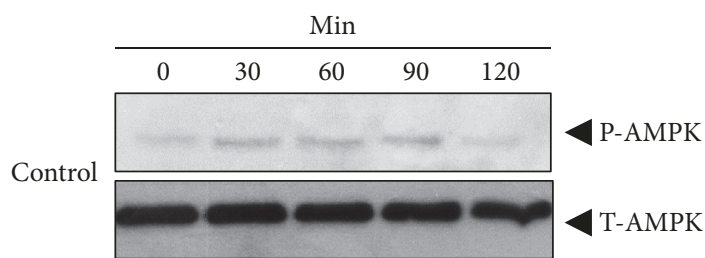
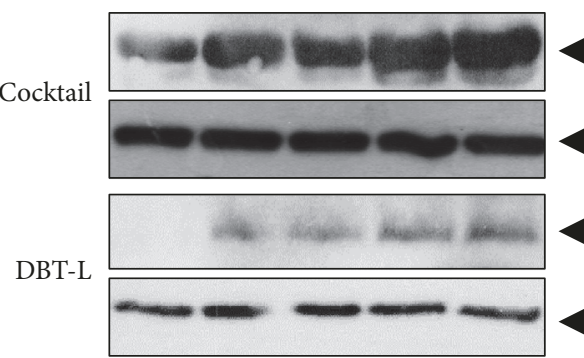

P-AMPK

T-AMPK

P-AMPK

DBT-H

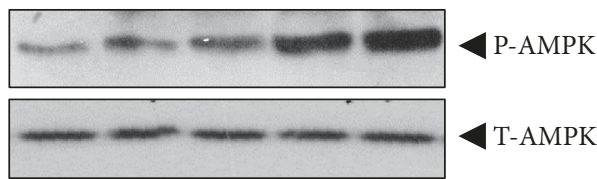

(a)

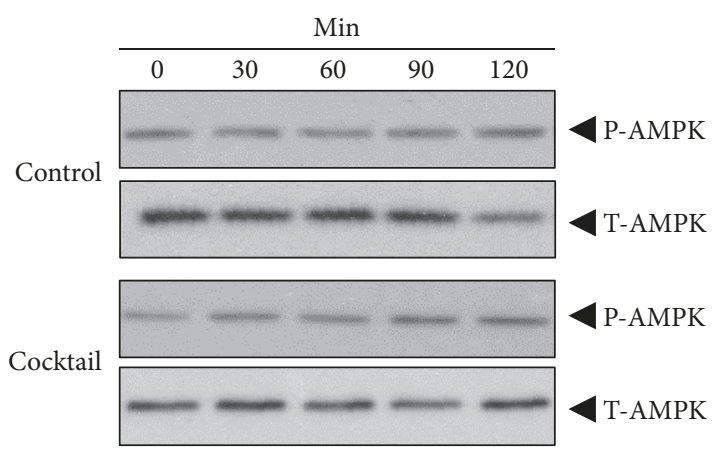

DBT-H

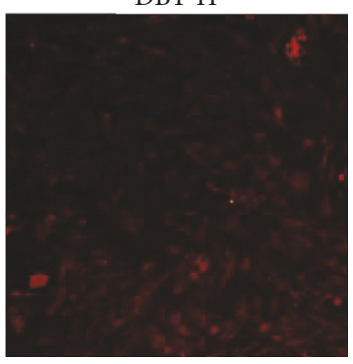

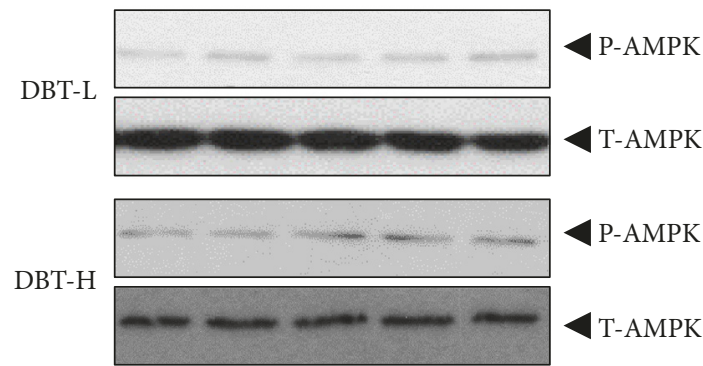

(b)

Figure 5: $D B T_{1155}$ stimulates $C a^{2+}-A M P K$ pathway. 3T3-L1 adipocytes were pretreated with medium (a) or BAMPTA-AM (10 $\mu$ M) (b) for 3 hours and then were labeled with fluorescent $\mathrm{Ca}^{2+}$ indicator Fluo- $4 \mathrm{AM}$ for half an hour. Fluorimetric measurement was performed after the treatment of different concentrations of $\mathrm{DBT}_{1155}$ decoctions, as in Figure 2. A23187 (100 nM) served as a control. The amounts of Ca ${ }^{2+}$ were evaluated by measuring the fluorescence intensity (left panel). Micrographs were taken by a confocal microscope; Bar $=100 \mu \mathrm{m}$. Differentiated cells were subjected to the phosphorylation assay. Phospho-AMPK (P-AMPK, $\sim 60 \mathrm{kDa}$ ) and total AMPK (T-AMPK, $60 \mathrm{kDa}$ ) were revealed by using specific antibodies (right panel). Representative photos were shown, $n=4$.

could be significantly suppressed by the $\mathrm{Ca}^{2+}$ chelator; hence, the abovementioned data strongly supported that this conventional herbal formula reduced obesity by a $\mathrm{Ca}^{2+}-\mathrm{AMPK}$ signaling.

Obesity posts a great challenge on body health in a variety of ways, including high blood pressure and cholesterol, cardiovascular diseases, type II diabetes, and musculoskeletal discomfort [44]. The antiobesity functions of $\mathrm{DBT}_{1155}$ were never reported and, therefore, we believe this could be a significant breakthrough for further study. The aim here is to reveal TCM formulae that could be used for obesity treatment. Although our in vitro data suggest this herbal 

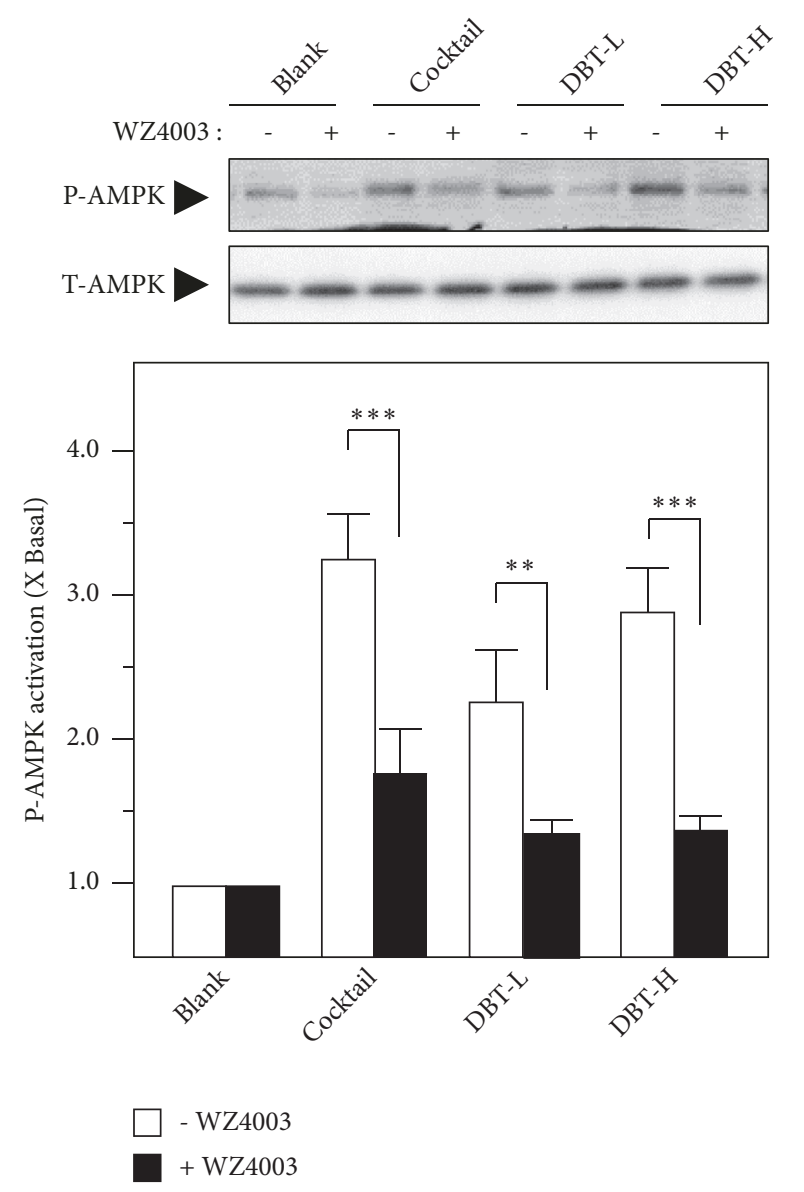

FIGURE 6: WZ4003 suppresses AMPK phosphorylation. 3T3-L1 adipocytes were pretreated with medium or WZ4003 (100 nM) for 3 hours and then subjected to the phosphorylation assay. The treatment of different concentrations of $\mathrm{DBT}_{1155}$ decoctions was as in Figure 2. Phospho-AMPK (P-AMPK, $\sim 60 \mathrm{kDa}$ ) and total AMPK (T-AMPK, $\sim 60 \mathrm{kDa}$ ) were revealed by using specific antibodies (upper panel). Quantification of protein expression was calculated by a densitometer (lower panel). Data were expressed as mean \pm SEM as compared with control, setting as 1 here, where $n=3 ; p<$ $0.05(*) ; p<0.01(* *) ; p<0.001(* * *)$.

formula possesses antiobesity functions via accelerating WAT browning and lipid catabolism, the in vivo experiments are indispensable as to further confirm the functions.

\section{Data Availability}

The data used to support the findings of this study are included within the article.

\section{Conflicts of Interest}

The authors declare that there is no conflict of interests regarding the publication of this paper.

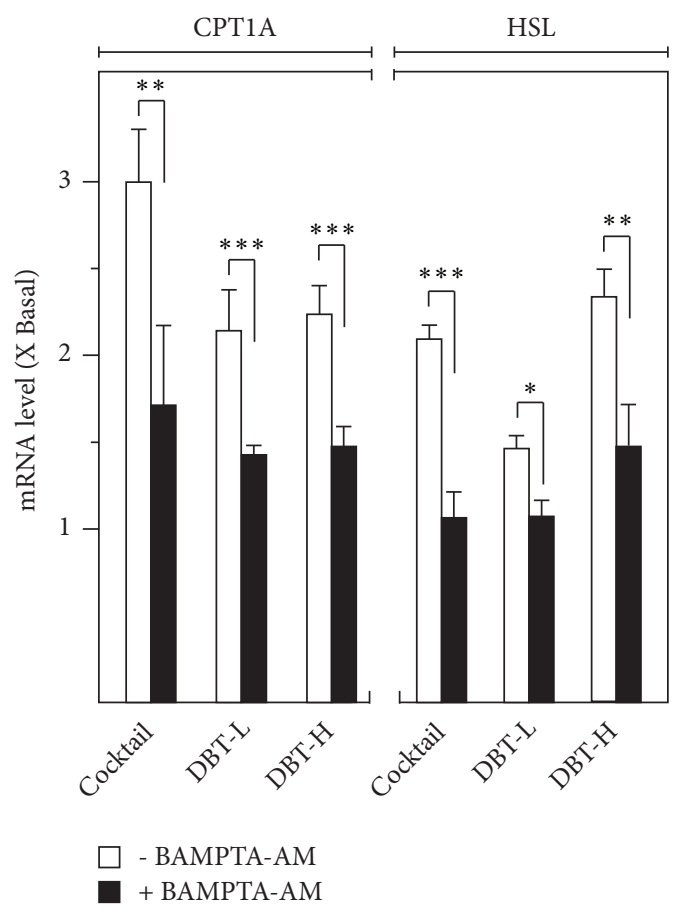

FIGURE 7: DBT enhances fatty acid catabolism. 3T3-L1 adipocytes were cultured for 10 days of differentiation. Then cocktail or different concentrations of $\mathrm{DBT}_{1155}$ with/without cotreatment of BAMPTAAM $(10 \mu \mathrm{M})$ were applied for another 3 days, as in Figure 2. Total RNAs were isolated and reverse-transcribed to cDNA for PCR analysis. The transcriptional levels of CPT1A and HSL were determined by the Ct-value method and normalized by $18 \mathrm{~S}$ rRNA. Data were expressed as mean \pm SEM as compared with control, setting as 1 here, where $n=3 ; p<0.05(*) ; p<0.01(* *) ; p<0.001$ $(* * *)$.

\section{Acknowledgments}

This study is supported by Research Fund of Zunyi Medical University for the Doctoral Program (F-937), NNSF of Guangdong (2018A030307074), Science and Technology Planning Project of Guangdong (2014A020221058), China, and Shenzhen Science and Technology Committee Research Grant (ZDSYS201707281432317; JCYJ20180214151016621; JCYJ20170413173747440; JCYJ 20160229205812004; JCYJ20160229210027564; CKFW2016082916015476).

\section{Supplementary Materials}

Supplementary Figure 1. Cell viability under the treatment of DBT1155. Supplementary Figure 2. ASR, AR, and JF show insignificant inhibition of lipid accumulation. Supplementary Table 1 Calibration curves, LOD and LOQ in HPLC analysis. (Supplementary Materials)

\section{References}

[1] D. W. Haslam and W. P. T. James, “Obesity," The Lancet, vol. 366, no. 9492, pp. 1197-1209, 2005. 
[2] P. Hossain, B. Kawar, and M. El Nahas, "Obesity and diabetes in the developing world-a growing challenge," The New England Journal of Medicine, vol. 356, no. 3, pp. 213-215, 2007.

[3] F. Bustamante, V. O. Brunaldi, W. M. Bernardo et al., "Obesity treatment with botulinum toxin-A is not effective: a systematic review and meta-analysis," Obesity Surgery, vol. 27, no. 10, pp. 2716-2723, 2017.

[4] K. D. Hoerster, S. Wilson, K. M. Nelson, G. E. Reiber, and R. M. Masheb, "Diet quality is associated with mental health, social support, and neighborhood factors among Veterans," Eating Behaviors, vol. 23, pp. 168-173, 2016.

[5] R. Fogari and A. Zoppi, "Effect of antihypertensive agents on quality of life in the elderly," Drugs \& Aging, vol. 21, no. 6, pp. 377-393, 2004.

[6] Y. Nishimoto and Y. Tamori, "CIDE family-mediated unique lipid droplet morphology in white adipose tissue and brown adipose tissue determines the adipocyte energy metabolism," Journal of Atherosclerosis and Thrombosis, vol. 24, no. 10, pp. 989-998, 2017.

[7] A. Guilherme, D. J. Pedersen, E. Henchey et al., "Adipocyte lipid synthesis coupled to neuronal control of thermogenic programming," Molecular Metabolism, vol. 6, no. 8, pp. 781-796, 2017.

[8] A. Ghasemi and S. Jeddi, "Anti-obesity and anti-diabetic effects of nitrate and nitrite," Nitric Oxide: Biology and Chemistry, vol. 70, pp. 9-24, 2017.

[9] J. Lone, J. H. Choi, S. W. Kim, and J. W. Yun, "Curcumin induces brown fat-like phenotype in 3T3-L1 and primary white adipocytes," The Journal of Nutritional Biochemistry, vol. 27, pp. 193-202, 2016.

[10] L. M. Acevedo, A. I. Raya, R. Ríos, E. Aguilera-Tejero, and J.L. L. Rivero, "Obesity-induced discrepancy between contractile and metabolic phenotypes in slow- and fast-twitch skeletal muscles of female obese Zucker rats," Journal of Applied Physiology, vol. 123, no. 1, pp. 249-259, 2017.

[11] M. T. Nakamura, B. E. Yudell, and J. J. Loor, "Regulation of energy metabolism by long-chain fatty acids," Progress in Lipid Research, vol. 53, no. 1, pp. 124-144, 2014.

[12] L. Zhang, C. Virgous, and H. Si, "Ginseng and obesity: observations and understanding in cultured cells, animals and humans," The Journal of Nutritional Biochemistry, vol. 44, pp. 1-10, 2017.

[13] N. Sun, T. Wu, and C. Chau, "Natural dietary and herbal products in anti-obesity treatment," Molecules, vol. 21, no. 10, p. E1351, 2016.

[14] M. S. Mansour, Y.-M. Ni, A. L. Roberts, M. Kelleman, A. Roychoudhury, and M.-P. St-Onge, "Ginger consumption enhances the thermic effect of food and promotes feelings of satiety without affecting metabolic and hormonal parameters in overweight men: a pilot study," Metabolism - Clinical and Experimental, vol. 61, no. 10, pp. 1347-1352, 2012.

[15] R. H. Mahmoud and W. A. Elnour, "Comparative evaluation of the efficacy of ginger and orlistat on obesity management, pancreatic lipase and liver peroxisomal catalase enzyme in male albino rats," European Review for Medical and Pharmacological Sciences, vol. 17, no. 1, pp. 75-83, 2013.

[16] G. Saravanan, P. Ponmurugan, M. A. Deepa, and B. Senthilkumar, "Anti-obesity action of gingerol: effect on lipid profile, insulin, leptin, amylase and lipase in male obese rats induced by a high-fat diet," Journal of the Science of Food and Agriculture, vol. 94, no. 14, pp. 2972-2977, 2014.

[17] V. Ebrahimzadeh Attari, A. Malek Mahdavi, Z. Javadivala, S. Mahluji, S. Zununi Vahed, and A. Ostadrahimi, "A systematic review of the anti-obesity and weight lowering effect of ginger (Zingiber officinale Roscoe) and its mechanisms of action," Phytotherapy Research, vol. 32, no. 4, pp. 577-585, 2018.

[18] Y. Zhao and Z.-Y. Chen, "Roles of spicy foods and their bioactive compounds in management of hypercholesterolemia," Journal of Agricultural and Food Chemistry, vol. 66, no. 33, pp. 86628671, 2018.

[19] M. Lu, Y. Cao, J. Xiao, M. Song, and C. Ho, "Molecular mechanisms of the anti-obesity effect of bioactive ingredients in common spices: a review," Food \& Function, vol. 9, no. 9, pp. 4569-4581, 2018.

[20] T. T. X. Dong, K. J. Zhao, Q. T. Gao et al., "Chemical and biological assessment of a Chinese herbal decoction containing Radix Astragali and Radix Angelicae Sinensis: determination of drug ratio in having optimized properties," Journal of Agricultural and Food Chemistry, vol. 54, no. 7, pp. 2767-2774, 2006.

[21] W. L. Zhang, K. Y.-Z. Zheng, K. Y. Zhu et al., "Chemical and biological assessment of Angelica herbal decoction: comparison of different preparations during historical applications," Phytomedicine, vol. 19, no. 11, pp. 1042-1048, 2012.

[22] A. G. Gong, N. Li, K. Lau et al., "Calycosin orchestrates the functions of Danggui Buxue Tang, a Chinese herbal decoction composing of Astragali Radix and Angelica Sinensis Radix: an evaluation by using calycosin-knock out herbal extract," Journal of Ethnopharmacology, vol. 168, pp. 150-157, 2015.

[23] C. T. Lam, P. H. Chan, P. S. Lee et al., "Chemical and biological assessment of Jujube (Ziziphus jujuba)-containing herbal decoctions: induction of erythropoietin expression in cultures," Journal of Chromatography B, vol. 1026, pp. 254-262, 2016.

[24] A. J. Guo, R. C. Choi, A. W. Cheung et al., "Stimulation of Apolipoprotein A-IV expression in Caco-2/TC7 enterocytes and reduction of triglyceride formation in 3T3-L1 adipocytes by potential anti-obesity Chinese herbal medicines," Chinese Medicine, vol. 4, no. 1, p. 5, 2009.

[25] T. C. Bargut, V. Souza-Mello, M. B. Aguila, and C. A. Mandarimde-Lacerda, "Browning of white adipose tissue: lessons from experimental models," Hormone Molecular Biology and Clinical Investigation, vol. 31, no. 1, 2017.

[26] P. Walle, M. Takkunen, V. Männistö et al., "Alterations in fatty acid metabolism in response to obesity surgery combined with dietary counseling," Nutrition \& Diabetes, vol. 7, no. 9, p. e285, 2017.

[27] S. Kim, S. Cheong, A. Chou, and R. Gonzalez, "Engineered fatty acid catabolism for fuel and chemical production," Current Opinion in Biotechnology, vol. 42, pp. 206-215, 2016.

[28] W.-L. Zhang, L. Zhu, and J.-G. Jiang, "Active ingredients from natural botanicals in the treatment of obesity," Obesity Reviews, vol. 15, no. 12, pp. 957-967, 2014.

[29] A. J. Silvester, K. R. Aseer, and J. W. Yun, "Dietary polyphenols and their roles in fat browning," The Journal of Nutritional Biochemistry, vol. 64, pp. 1-12, 2019.

[30] A. J. Akinyemi, G. R. Thomé, V. M. Morsch et al., "Effect of ginger and turmeric rhizomes on inflammatory cytokines levels and enzyme activities of cholinergic and purinergic systems in hypertensive rats," Planta Medica, vol. 82, no. 7, pp. 612-620, 2016.

[31] N. Ning and J. J. Han, "Advances in studies on active components of reducing blood lipid in traditional Chinese medicine," Asia-Pacific Traditional Medicine, vol. 9, pp. 76-79, 2013.

[32] G. A. Mohamed, S. R. Ibrahim, E. S. Elkhayat, and R. S. El Dine, "Natural anti-obesity agents," Bulletin of Faculty of Pharmacy, Cairo University, vol. 52, no. 2, pp. 269-284, 2014. 
[33] H. Kubota, R. Morii, A. Kojima-Yuasa, X. Huang, Y. Yano, and I. Matsui-Yuasa, "Effect of Zizyphus jujuba extract on the inhibition of adipogenesis in 3T3-L1 preadipocytes," American Journal of Chinese Medicine, vol. 37, no. 3, pp. 597-608, 2009.

[34] X. L. Zhong, J. L. Ding, and D. S. Luo, "Progress on blood lipid regulating active ingredients from traditional Chinese medicine," Food Drug, vol. 11, pp. 449-451, 2010.

[35] M. Zhang, C. P. Zhu, C. L. Shi, J. X. Xiao, and S. H. Zhang, "Extraction, isolation of Lycium barbarum polysaccharide-4 and its role in weight loss obesity mice on female hypothalamus injury," Nutrition and Health, vol. 24, pp. 114-117, 2003.

[36] R. L. C. Hoo, J. Y. L. Wong, C. F. Qiao, A. Xu, H. X. Xu, and K. S. L. Lam, "The effective fraction isolated from Radix Astragali alleviates glucose intolerance, insulin resistance and hypertriglyceridemia in $\mathrm{db} / \mathrm{db}$ diabetic mice through its antiinflammatory activity," Journal of Nutrition and Metabolism, vol. 7, 2010.

[37] Y. Pan, D. Zhao, N. Yu et al., "Curcumin improves glycolipid metabolism through regulating peroxisome proliferator activated receptor $\gamma$ signalling pathway in high-fat diet-induced obese mice and 3T3-L1 adipocytes," Royal Society Open Science, vol. 4, no. 11, p. 170917, 2017.

[38] T. Jin, Z. Song, J. Weng, and I. G. Fantus, "Curcumin and other dietary polyphenols: potential mechanisms of metabolic actions and therapy for diabetes and obesity," American Journal of Physiology-Endocrinology and Metabolism, vol. 314, no. 3, pp. E201-E205, 2018.

[39] A. Shehzad, M. Qureshi, M. N. Anwar, and Y. S. Lee, "Multifunctional curcumin mediate multitherapeutic effects," Journal of Food Science, vol. 82, no. 9, pp. 2006-2015, 2017.

[40] Y. Zhao, B. Chen, J. Shen et al., "The beneficial effects of quercetin, curcumin, and resveratrol in obesity," Oxidative Medicine and Cellular Longevity, vol. 2017, Article ID 1459497, 8 pages, 2017.

[41] J. Gautam, V. Khedgikar, P. Kushwaha et al., "Formononetin, an isoflavone, activates AMP-activated protein kinase $\beta$-catenin signalling to inhibit adipogenesis and rescues $\mathrm{C} 57 \mathrm{BL} / 6$ mice from high-fat diet-induced obesity and bone loss," British Journal of Nutrition, vol. 117, no. 5, pp. 645-661, 2017.

[42] O. H. Kang, S. B. Kim, Y. S. Seo et al., "Curcumin decreases oleic acid-induced lipid accumulation via AMPK phosphorylation in hepatocarcinoma cells," European Review for Medical and Pharmacological Sciences, vol. 17, no. 19, pp. 2578-2586, 2013.

[43] S. Lv, X. Qiu, J. Li et al., "Glucagon-induced extracellular cAMP regulates hepatic lipid metabolism," Journal of Endocrinology, vol. 234, no. 2, pp. 73-87, 2017.

[44] N. Lv, K. M. J. Azar, L. G. Rosas, S. Wulfovich, L. Xiao, and J. $\mathrm{Ma}$, "Behavioral lifestyle interventions for moderate and severe obesity: a systematic review," Preventive Medicine, vol. 100, pp. 180-193, 2017. 


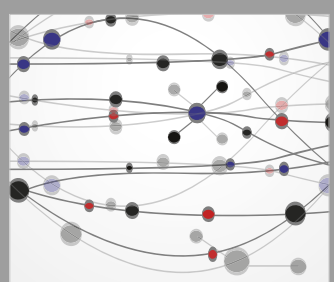

The Scientific World Journal
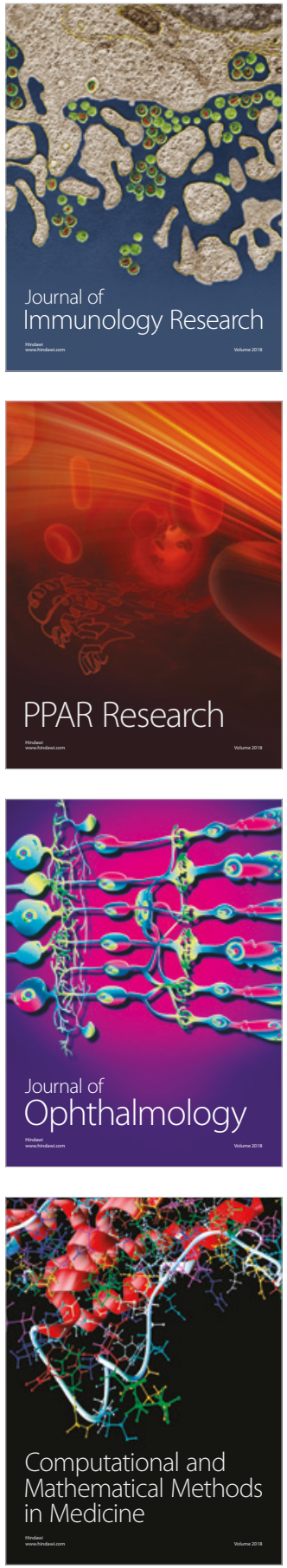

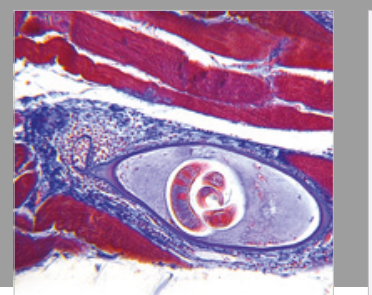

Gastroenterology Research and Practice

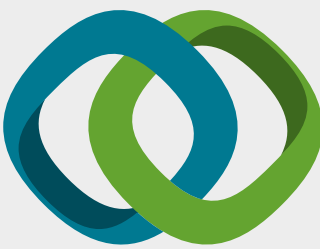

\section{Hindawi}

Submit your manuscripts at

www.hindawi.com
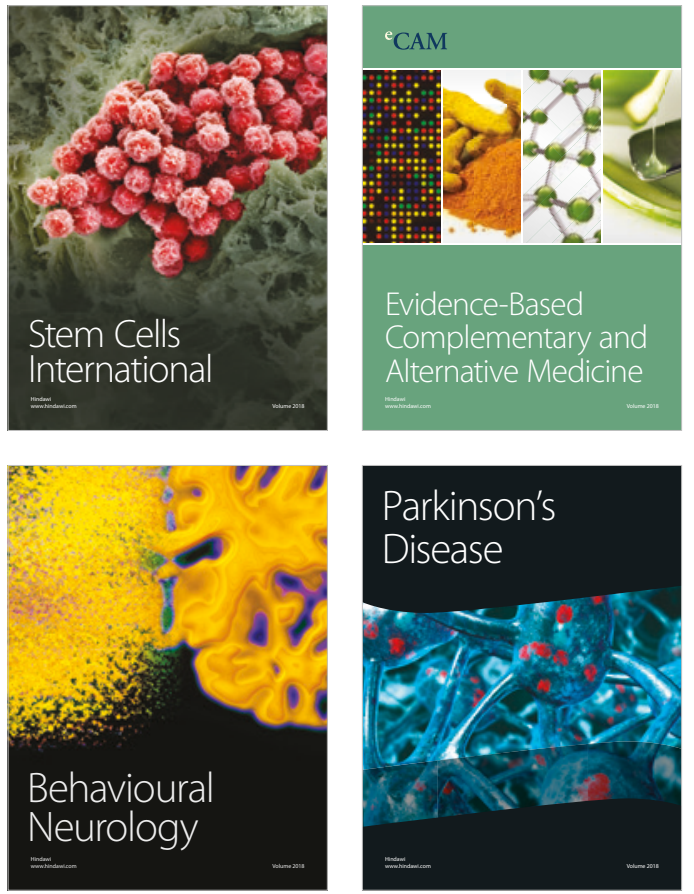

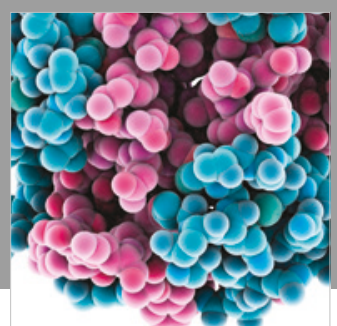

ournal of

Diabetes Research

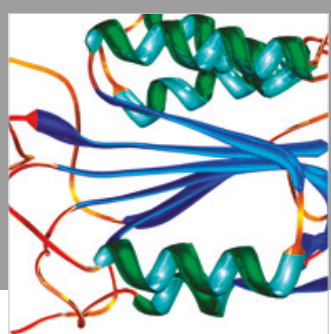

Disease Markers
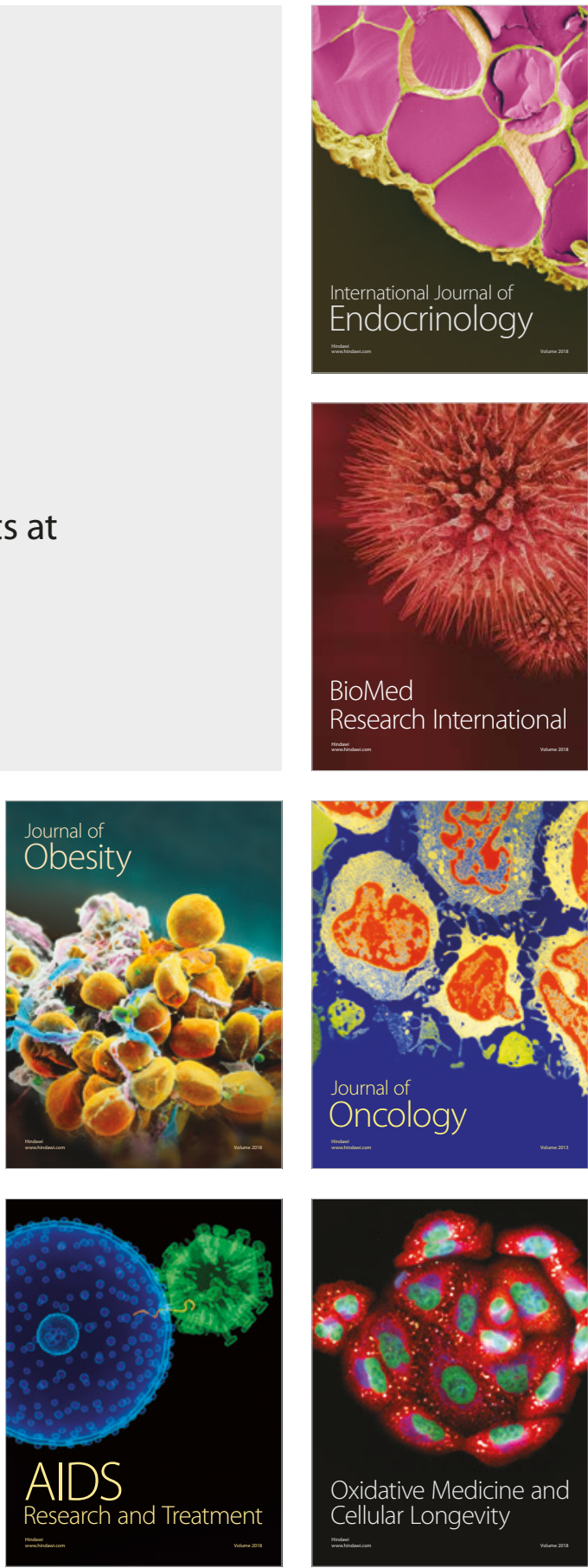